\title{
CIUDADANOS O CONSUMIDORES OFERTA Y DEMANDA EN LA FORMACIÓN DEL CUERPO INFANTIL
}

Mariano Narodowski*

RESUMEN - El objetivo de este artículo es analizar la cuestión de la ciudadanía a partir del estudio de los programas de "elección libre de escuelas" (school choice) desde una perspectiva no ideológica. A partir de una concepción igualitarista radical se analizan los cambios en el proceso de escolarización y las posibilidades que se abren (y las amenazas que encierran) la desestatalización de la educación escolar y la ruptura del monopolio cultural del Estado en materia educativa.

PALAVRAS-CHAVE - cidadão, consumidores e formação do corpo infantil.

\begin{abstract}
The objective of this article is to analyze the matter of citizenship by means of a study of the school choice programs as seen from a non-ideological perspective. Changes in the schooling process, and the possibilities which are opened up (as well as the resulting threats) as a consequence of the privatization of school education and the breaking up of the cultural monopoly of the State in terms of education are analyzed from a radical egalitarian point of view. KEY WORDS - citizen, consumer, formation of the infantile body.
\end{abstract}

\section{Nuevas políticas educativas}

La discusión en torno a la cuestión de la ciudadania merece analizarse en relación a programas concretos de política educativa que predican la necesidad de reestablecer la capacidad de elección escolar como elemento central de una democratización efectiva.

En los últimos lustros, en el ámbito de la política educacional latinoamericana se han levantado algunas voces a favor y contra la aplicación de programas de libre elección de escuelas (school choice) en la América Latina. Probablemente a causa de las propuestas de los organismos financieros internacionales como el Banco Mundial (West, 1996), de las repercusiones de la discusión académica internacional (Whitty, 1997) o las experiencias chilena o colombiana en materia de descentralización y subsidio a la demanda (OEI, 1993; 1995) el tema ha venido generando una interesante discusión en medios políticos y académicos.

* Doctor en Educación, profesor titular de la Universidad Nacional de Quilmes/Argentina. 
Esquemáticamente (y resumiendo la disimil experiencia de países que aplican estas políticas como Inglaterra, Suecia, Polonia, Nueva Zelanda y algunos estados norteamericanos) los programas de school choice consisten en el aporte estatal de fondos públicos a la demanda educativa (es decir, a los padres de un alumno) y no, como es habitual en la mayoría de los sistemas educativos occidentales, a la oferta escolar, en la que el Estado dota de fondos a las escuelas y paga el salario a los docentes. En el caso de los programas de school choice, este aporte estatal a la demanda se brinda usualmente por medio de vouchers o vales que reciben los padres y que fijan el valor económico del curso al cual el alumno puede acceder por su edad. Estos vales pueden ser canjeados por el servicio educativo a elección de la familia del estudiante.

El subsidio a la demanda permite así la libre elección de escuela y, como contrapartida, estimula a que las instituciones escolares construyan un determinado perfil que sea competitivo a fin de captar a la demanda, puesto que su éxito o su quiebra van a depender del número de vales que logren acumular. Se conforma asi un mercado (Chubb and Moe, 1990) o un "quasi mercado" (Whitty, 1997) educativo.

En la experiencia internacional, los programas de elección de escuela no están atados a la dicotomía estatal-privado. Los Estados Unidos son un ejemplo de diversidad ya que si bien algunos gobernadores estatales (republicanos o demócratas) censuran la aplicación de estas políticas, en otros estados permiten la elección subsidiada de escuelas privadas (por medio de deducciones impositivas) mientras que otros solo aplican el vale entre las escuelas públicas (cfr. Shokraii y Youssef, 1998). Este sistema de libre elección de escuela tampoco implica "municipalizar" la gestión o el gobierno de la educación puesto que la unidad de cálculo del subsidio y de distribución del mismo puede ser, indistintamente, local, provincial o federal.

El school choice, aparte de sus características económicas y de la creación de mercados o quasi mercados, permite la formalización de mayor autonomía institucional en los proyectos pedagógicos de las escuelas puesto que la propia naturaleza del programa debería generar, según la propuesta de sus principales defensores (Chubb and Moe, 1993) un enorme abanico de diversidad institucional. Esto se expresa, especialmente en el caso de los Estados Unidos, en la aparición de un sinnúmero de posibilidades de escuelas estatales: las denominadas magnet school (escuelas imán) que intentan atraer al alumnado por medio de programas educativos especiales y diferenciados (como por ejemplo, educación por el arte) y en las charter school: emprendimientos conformados por grupos de maestros y padres en base a la posibilidad de captar vales o apoyo estatal directo para construir escuelas con un determinado perfil cultural, ideológico o religioso. Así es posible "chartear" una escuela si se consigue atraer los vales necesarios para conformarlá.

Queda claro pues que la elección libre de escuela, como afirman Cosse, Morduchowicz y Raschia (1997), no constituye de manera alguna una estrategia de financiamiento o de desfinanciamiento de la educación escolar sino más bien una metodología para asignar recursos y gastar dinero público. Por supuesto, la diver- 
sificación de la oferta educativa y la consiguiente desburocratización de su funcionamiento como único medio de lograr perfiles institucionales y así atraer a la demanda también puede implicar, aunque no necesariamente, una ampliación de las posibilidades de autofinanciamiento por parte de cada unidad escolar.

En los países de América Latina, la estrategia de aplicación de programas de política educativa basados en la elección de escuela y en el subsidio a la demanda choca contra dos barreras. Una primer barrera de carácter histórica producto de una tradición de Estados fuertes que transfieren recursos financieros hacia una oferta escolar socialmente legitimada. Es que, durante décadas, la mayor parte de la oferta de educación escolar en los sistemas educativos latinoamericanos mantuvieron una gestión altamente centralizada (especialmente en lo atinente a las escuelas públicas). Los estudios clásicos de la política educativa de los ' 80 coincidieron en demostrar que la acción Estatal en el sostenimiento financiero directo de las escuelas parece haber constituido una de las principales causas para que la región incrementara notablemente el rendimiento de sus indicadores cuantitativos en cuanto a expansión y democratización de los sistemas educativos.

Una segunda barrera existente para la aplicación de programas de libre elección de escuelas es de carácter legal puesto que la elección paterna de la escuela a la que habrán de concurrir los respectivos hijos no constituyó un derecho tutelado por la mayoria de los textos constitucionales o las leyes de los países de América Latina.

\section{La efectiva elección de escuela}

A pesar de la existencia de barreras legales y de la presencia de una fuerte discusión jurídica y política acerca de la cuéstión de la demanda, en América Latina de todos modos ya existe el school choice y no solamente en el caso colombiano o chileno sino que se trata de una práctica social fuertemente extendida en la región. Vale la pena observar que la elección de escuelas está reservada fundamentalmente para las clases o grupos sociales que poseen los medios de dispensar la asistencia estatal en materia de escuela pública y así sustentar la elección de la educación de sus propios hijos en las instituciones escolares que ellos determinan como más convenientes para sus aspiraciones intelectuales, culturales, ideológicas, religiosas y/o sociales.

Claro está que esto no significa que exista "subsidio estatal a la demanda educativa" en términos estrictos, puesto que el esfuerzo financiero estatal está puesto en el aporte de recursos a la oferta, tanto a la del sector estatal como a la del sector privado, aunque a esta en una medida mucho menor. Sin embargo, algunos grupos sociales poseen capacidad de elegir escuela sin contar con el apoyo financiero estatal directo: se trata de formas de presión social que resuelven el problema de la satisfacción de las necesidades de la demanda sin que la misma sea subsidiada en forma directa. ¿Quiénes y cómo elaboran esas estrategias por medio de las cuales, en un contexto de regulación estatal de la oferta, pueden igualmente elegir escuela para sus hijos? 
En América Latina, es posible detectar dos grandes grupos sociales capaces de elegir escuela. Unos respaldan su decisión en su capacidad económica (directa o indirecta) mientras que otros la respaldan básicamente en su capacidad de presión política. El primer grupo está identificado con la elección de escuelas en el sector privado mientras que el segundo con el sector estatal.

Cuando el respaldo para la elección es de carácter económico, los padres que se encuentran en situación de sostener económicamente su decisión de elegir escuela son capaces de absorber un doble impacto: pagan sus impuestos y contribuyen así al sostenimiento del sistema educativo en su conjunto y a la vez efectúan erogaciones directas al contratar el servicio escolar privado. En algunos países, existen igualmente exenciones impositivas que atenúan este impacto tributario.

En otros casos también pertenecientes a este primer grupo, la elección de escuela está sostenida económicamente de modo indirecto y parece estar reservada para aquellos sectores de la población que aunque carentes de recursos económicos están dispuestos a optar por una educación religiosa (en América Latina, se trata de establecimientos mayoritariamente católicos) en escuelas que, con o sin el apoyo financiero del Estado o de las propias instituciones confesionales, pueden ofrecer un servicio privado gratuito o a un precio muy accesible para estos sectores.

El segundo grupo de electores de escuela se corresponde con algunos grupos sociales que pueden optar por escuelas públicas por ellos consideradas de alta calidad y/o prestigio social aunque las mismas no se correspondan con el radio o sector en el que habitan. Para lograr la anhelada vacante parecen poner en juego sus influencias políticas y/o corporativas, el apoyo económico a las asociaciones de padres o el prestigio personal o profesional del padre o la madre. Consiguen de esta manera una inscripción escolar para sus hijos utilizando circuitos informales procediendo por medio de una suerte de "contrabando" de influencias y presiones que permiten eludir la hiperregulación estatal en materia de elección de escuelas públicas.

En resumen, en América Latina la elección libre de escuela existe aún en los contextos de mayor regulación pero la misma está restringida a los siguientes grupos sociales: pueden elegir escuela quienes tienen capacidad pecuniaria de absorber una doble imposición tributaria, pueden elegir escuela quienes aceptan enviar a sus hijos a escuelas confesionales gratuitas o de muy bajo precio (con independencia del poder adquisitivo) y, finalmente, pueden elegir escuela quienes tienen capacidad de ejercer poder político, económico o profesional para convenir con la dirección de una escuela pública la inscripción de su hijo.

Solamente estos grupos sociales están en condiciones de ejercer su capacidad de elección respecto de su preferencia por lo laico o lo confesional, lo público o lo privado, la educación bilingüe, la formación en los deportes, o la educación por el arte, entre otras. El resto, la gran mayoría de los padres y alumnos debe resignarse a esperar que lo que haga la escuela pública a la que debe concurrir se adecue a sus intereses y opciones culturalés. Y si no se adecua (porque su oferta es de bajo 
nivel, porque los docentes ganan poco, porque ofrece una opción cultural que no es de su preferencia, etc.) a esos padres y a esos alumnos solo les resta el camino de la protesta política, porque, por ser pobres, no tienen otra opción que aquella que el monopolio estatal sobre la escuela pública les ofrece. Y si son migajas de educación lo que se les ofrece, a esas migajas se tienen que resignar.

\section{Cambios en la formación del cuerpo infantil}

El objetivo de nuestro punto de vista no pretende un posicionamiento ideologista para intentar eliminar la educación escolar de gestión estatal ni la de gestión privada. No interesa aquí aportar slogans de derecha ("competencia en el sistema educativo") o de pseudo izquierda ("macdonaldización de la escuela"). Al contrario de Coulson (1996) en los sistemas educativos latinoamericanos no se encuentran elementos que prueben que la expansión de la matrícula a lo largo del siglo XX fuera producto de la acción de los particulares en el contexto de un mercado libre sino, al contrario, los historiadores de la educación de diversas tendencias historiográficas parecen coincidir que el Estado fue el agente principal en esta política. Pero estos estudios también confirman que la regulación estatal de la oferta escolar tampoco resolvió el problema de la democratización del acceso a una educación escolar de calidad.

En otras palabras, el Estado ya ni consigue prometer lo que hasta ahora no ha cumplido. Los datos aportados inducen a pensar en una suerte de decadencia estatal en materia de cobertura de la matrícula a lo que se le suma la burocratización de esa oferta y la desregulación de la oferta privada, más proclive a adaptarse a las necesidades de la demanda.

Por este motivo, parece necesario en cualquier proceso de democratización de la educación, una política que sea capaz de restituirle a las escuelas estatales las mismas herramientas con las que cuenta la escuela privada, montando un mecanismo de competencia y calidad sobre el bien público y brindándoles poder a los padres para que decidan sobre la educación de sus hijos: a todos los padres y no solamente a aquellos de clase media y clase alta a los que las leyes del mercado les permiten elegir y pagar su escuela.

No se trata de sostener que la libre elección de escuela traiga de suyo un aumento en la calidad del servicio educativo, entendida esta en los más crudos niveles de resultados a test de rendimiento en los aprendizajes. Este, en realidad, es el tema más controvertido ya que Chubb y Moe (1991) intentaron demostrar una fuerte asociación entre los programas de libre elección escolar y un aumento en la performance de los alumnos incluidos en este programa. En la literatura especializada (Chubb y Moe, 1990; Smith y Meier, 1995) no existe acuerdo al respecto aunque recientes análisis de casos tienden a mostrar que la asociación entre elección de escuelas y rendimiento se fortalece a medida que desciende el origen socioeconómico de la matrícula y que se consolidan los procesos de autonomización en la construcción de un proyecto pedagógico de la institución que, a fin de captar demanda, satisfaga sus necesidades educativas (Greene, Howell y Peterson, 1997). 
En realidad la resolución del problema dispensa de ideologismos o fundamentalismos: el problema es si el Estado tiene todavía capacidad de formar al cuerpo infantil o si las familias pueden recuperar esa función que perdieron con la creación de los sistemas educativos nacionales. La respuesta no es sencilla ni generalizable puesto que sistemas educativos altamente centralizados y con poca capacidad de demanda por parte de sus actores (Alemania, Francia y Japón, por ejemplo) consiguen los más altos estándares de calidad. Sin embargo, parece ser que en América Latina, los sectores sociales con capacidad de hacer valer su elección consiguen que la escuela privada satisfaga sus necesidades de educación escolar para sus hijos.

Es evidente que un amplio campo de discusión académica y política se abre frente a esta perspectiva, siempre y cuando la misma pueda acometerse revisando algunos ideologismos.

El primero, sin duda, es suponer que el sistema de elección de escuelas es esencialmente una estrategia de ajuste presupuestario. Este argumento es difícil de sostener en la mayor parte de los países latinoamericanos en la medida que el colapso presupuestario en el que se hallan los sistemas educativos se ubica en el contexto de políticas estatales de apoyo a la oferta. Sin embargo, también es posible demostrar lo herrado de esta conjetura a partir de estudios empíricos: para el caso norteamericano, algunos estudios han constatado un aumento considerable de costos en los programas de elección de escuelas (The Carnegie Foundation, 1992; Doerr, Menendez y Swomley, 1996).

El segundo consiste en la necesidad de estudiar la perspectiva de la demanda. Sería necesario determinar si la matrícula de escuela estatal es el resultado de la incapacidad económica o política de elegir escuela o, al contrario, producto de una decisión sustentable por parte de los padres. Y, al mismo tiempo, determinar el sentido de la elección de escuela privada en el contexto de la satisfacción del cliente.

Esto no significa suponer un desplazamiento de una visión de ciudadanía a una de consumidores acríticos para el caso de los tomadores del servicio educativo. Esta dicotomía resulta sospechosa en un escenario en el que se permite a los sectores sociales con mayor poder adquisitivo elegir el sesgo pedagógico y cultural de la escuela de sus hijos (o sea, los ricos pueden consumir) y se plantea para los pobres que sean lúcidos representantes de una conciencia ciudadana crítica y transformadora. En nuestra opinión, este planteo no es más que un enfoque desgarradoramente cínico respecto de las posibilidades de otra forma de educar a la infancia.

A partir de los trabajos de Gintis (1994 y 1995) queda claro que la pelea por un igualitarismo radical requiere de respuestas imaginativas, capaces de desmontar el aparato estatal de desigualdad y de imposición cultural montado a lo largo de décadas de educación escolar em América Latina. 


\section{Referências bibliográficas}

ASCHER, Carol, FRUCHTER, Norm, BERNE, Robert. Hard lessons. Public schools and privatization. New York: Twentieth Century Fund., 1996.

CHUBB, John E., MOE, Terry M. A lesson in School Reform from Great Britain. Washington, DC: The Brookings Institution, 1992. . Politics, markets, and America's schools. Washington, DC: The Brookings Institution, 1990.

DOERR, Edd, MENENDEZ, Albert J., SWOMLEY, John M. The case against School Vouchers. Prometheus Books. U.S.A., 1995.

ELSTER, Jon. Justicia local. De qué modo las instituciones distribuyen bienes escasos y cargas necesarias. Madrid: Gedisa, 1996.

FULLER, Bruce, ELMORE, Richard F. Who chooses? Who loses. Teacher College. U.S.A., 1996.

GINTIS, Herbert. "The political economy of school choice". Teachers College Record, v. 96, n. 3, 1995.

GREENE, Jay P., HOWELL, William G., PETERSON, Paul E. An evaluation of the Cleveland scholarship program. Cambridge: Harvard University, 1997.

JACOBSON, Susan. Getting the public school you want. San Francisco: Publishing 20/20, 1996.

HENIG, Jeffrey R. Rethinking school choice. Princeton, 1994.

OEI, Sistemas Educativos Nacionales. Chile, Madrid, 1993.

OEI, Sistemas Educativos Nacionales. Colombia, Madrid, 1995.

SHOKRAI, Nina H., YOUSSEF, Sarah E. School choice programs: what's happening in the States. Washington: The Heritage Foundation, 1998.

SMITH, Kevin B., MEIER, Kenneth J. The case against school choice. M. E. Sharpe. U.S.A., 1995. 